\title{
The offset-midpoint traveltime pyramid in 3D transversely isotropic media with a horizontal symmetry axis
}

\author{
Qi Hao ${ }^{1}$, Alexey Stovas ${ }^{1}$, and Tariq Alkhalifah ${ }^{2}$
}

\begin{abstract}
Analytic representation of the offset-midpoint traveltime equation for anisotropy is very important for prestack Kirchhoff migration and velocity inversion in anisotropic media. For transversely isotropic media with a vertical symmetry axis, the offset-midpoint traveltime resembles the shape of a Cheops' pyramid. This is also valid for homogeneous 3D transversely isotropic media with a horizontal symmetry axis (HTI). We extended the offset-midpoint traveltime pyramid to the case of homogeneous 3D HTI. Under the assumption of weak anellipticity of HTI media, we derived an analytic representation of the P-wave traveltime equation and used Shanks transformation to improve the accuracy of horizontal and vertical slownesses. The traveltime pyramid was derived in the depth and time domains. Numerical examples confirmed the accuracy of the proposed approximation for the traveltime function in 3D HTI media.
\end{abstract}

\section{INTRODUCTION}

In most cases, transversely isotropic media with a horizontal symmetry axis (HTI) media are caused by a system of parallel vertical circular ("penny-shaped") cracks embedded in an isotropic background (Tsvankin, 2001, pp. 12-13). The azimuthal characteristics of seismic waves in HTI media can help to identify the azimuth of fractures (e.g., Grechka and Tsvankin, 1999; Bakulin et al., 2000), which is important for oil and gas exploration in fractured reservoirs. The azimuthal variation of velocities influences traveltimes in HTI media.

For prestack phase-shift migration of common-midpoint (CMP) gathers, traveltime as an analytic function of offset and midpoint is given by a simple double-square-root (DSR) equation in homogeneous, isotropic media (Yilmaz, 2001, p. 638). Because the traveltime surface on the offset-midpoint plane from an image point is like a pyramid, Claerbout (1985, pp. 164-166) first names it as Cheops' pyramid. The traveltime pyramid is often used for prestack time migration (Yilmaz, 2001, pp. 725-728). However, it is difficult to obtain the analytic traveltime formulations in anisotropic media because the explicit relationship between group velocity and ray angle does not exist in anisotropic media. Even for transversely isotropic media with a vertical symmetry axis (VTI), the traveltime is often calculated numerically. Alkhalifah (2000b) derives the offsetmidpoint traveltime equation or Cheops' pyramid equation for VTI media under the assumption of weak anellipticity. Hao and Stovas (2013) extend the offset-midpoint traveltime equation to the case of 2D transversely isotropic media with a tilt symmetry axis (TTI).

The core of this paper is to propose an analytic traveltime approximation for prestack Kirchhoff migration in homogeneous 3D HTI media with the aid of the stationary phase method and the slowness surface rotation between HTI and VTI media. Although only the case of 3D homogeneous HTI media is discussed in this paper, the time-domain traveltime pyramid derived by us can apply to prestack Kirchhoff time migration by using the effective medium parameters. To implement the depth-domain traveltime pyramid for the prestack Kirchhoff depth migration in a vertically inhomogeneous medium, it can be assumed that this vertically inhomogeneous medium is locally "homogeneous" for each downward extrapolation step $\Delta z$. In each extrapolation step $\Delta z$, the traveltime is analytically calculated by means of our approach and wavefield migration is realized by the sum of the migration operator given by equation 1 over all traces recorded at the previous depth grid.

The paper is organized as follows: After this introduction, we will first derive the ray traveltime from the offset-midpoint phase-shift migration operator with the aid of the stationary phase method. We will then derive the analytic approximation for source and receiver

First presented at the SEG 81st Annual International Meeting. Manuscript received by the Editor 28 August 2013; revised manuscript received 15 September 2014; published online 30 December 2014.

${ }^{1}$ Norwegian University of Science and Technology, Department of Petroleum Engineering and Applied Geophysics, Trondheim, Norway. E-mail: qi.hao@ ntnu.no; alexey.stovas@ntnu.no.

${ }^{2}$ King Abdullah University for Science and Technology, Physical Science Division, Thuwal, Saudi Arabia. E-mail: tariq.alkhalifah@kaust.edu.sa.

(C) 2014 Society of Exploration Geophysicists. All rights reserved. 
slownesses by a combination of the slowness surface rotation between VTI and HTI media and the Shanks transformation (Bender and Orszag, 1978, pp. 369-375), from which we will obtain the traveltime pyramid in the depth and time domains. As a special case, the $\mathrm{P}$-wave reflection traveltime formulation for a horizontal reflector in 3D HTI media is further obtained by simplifying the time-domain traveltime pyramid. We will next illustrate the shape of the time-domain traveltime pyramid, the accuracy of the reflection traveltime formulation and the common-offset migration isochrones obtained from the depth-domain traveltime pyramid by some simple numerical examples. We will finally present our conclusions.

\section{THE STATIONARY PHASE METHOD}

In this section, we derive the exact expression for scattering ray traveltime in a homogeneous 3D HTI medium from the stationary phase approximation of wavefield extrapolation. According to equations A-24 and A-27 given in Appendix A, the single-trace response of the 3D prestack phase-shift migration defined in the halfoffset-midpoint domain for homogeneous anisotropic media reads

$$
\begin{aligned}
& P\left(x_{1}, x_{2}, h_{1}=0, h_{2}=0, z, t=0\right) \\
& =\iiint \int \tilde{P}\left(x_{1}^{0}, x_{2}^{0}, h_{1}^{0}, h_{2}^{0}, z\right. \\
& =0, \omega) \exp (i \omega T) d \omega d k_{h 1} d k_{h 2} d k_{x 1} d k_{x 2},
\end{aligned}
$$

where $T$ is the traveltime shift given by

$$
\begin{aligned}
T= & \left(q_{s}+q_{g}\right) z-2 p_{x 1}\left(x_{1}-x_{1}^{0}\right)-2 p_{x 2}\left(x_{2}-x_{2}^{0}\right) \\
& +2 p_{h 1} h_{1}^{0}+2 p_{h 2} h_{2}^{0}
\end{aligned}
$$

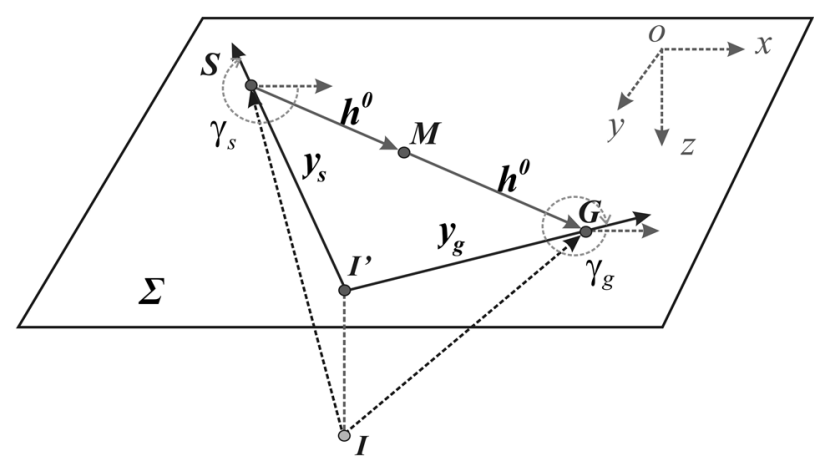

Figure 1. The geometric explanation of quantities in equations 1 and 2. In the Cartesian coordinate system (oxyz), the source $\mathbf{S}=\left(s_{1}, s_{2}, 0\right)$, the receiver $\mathbf{G}=\left(g_{1}, g_{2}, 0\right)$, and the midpoint $\mathbf{M}=$ $\left(x_{1}^{0}, x_{2}^{0}, 0\right)$ are located on the surface $\boldsymbol{\Sigma}$. The half-offset vector $\mathbf{h}^{0}=\left(h_{1}^{0}, h_{2}^{0}\right)$ equals a half of the distance vector from the source $\mathbf{S}$ to the receiver $\mathbf{G}$. The point $\mathbf{I}=\left(x_{1}, x_{2}, z\right)$ is an image point. The point $\mathbf{I}^{\prime}=\left(x_{1}, x_{2}, 0\right)$ is the projection of the image point $\mathbf{I}$ on the surface $\boldsymbol{\Sigma}$. The vector $\mathbf{y}_{s}=\left(y_{s 1}, y_{s 2}\right)$ denotes the lateral distance from the source $\mathbf{S}$ to the image point $\mathbf{I}$, and the vector $\mathbf{y}_{g}=\left(y_{g 1}, y_{g 2}\right)$ denotes the lateral distance from the receiver $\mathbf{G}$ to the image point I. $\gamma_{s}$ and $\gamma_{g}$ denote the azimuths of vectors $\mathbf{y}_{s}$ and $\mathbf{y}_{g}$, respectively. The seismic ray from the image point $\mathbf{I}$ to the source $\mathbf{S}$ has the source slowness $\left(p_{s 1}, p_{s 2},-q_{s}\right)$, and the seismic ray from the image point $\mathbf{I}$ to the receiver $\mathbf{G}$ has the receiver slowness $\left(p_{g 1}, p_{g 2},-q_{g}\right)$. These slownesses in the source-receiver domain can be converted to midpoint-half-offset domain through equation 3 . and $\tilde{P}$ and $P$ are seismic traces before prestack depth migration and the seismic image after prestack depth migration, respectively; subscripts 1 and 2 denote $x$ - and $y$-axes, respectively; $\left(x_{1}^{0}, x_{2}^{0}\right)$ is the midpoint position on acquisition surface; $\left(x_{1}, x_{2}, z\right)$ is the position of the image point; $\left(h_{1}^{0}, h_{2}^{0}\right)$ is the source-receiver half-offset; $q_{s}$ and $q_{g}$ are the vertical slowness components defined at source and receiver positions, respectively; $\left(p_{x 1}, p_{x 2}\right)=\left(k_{x 1} /(2 \omega), k_{x 2} /(2 \omega)\right)$ and $\left(p_{h 1}, p_{h 2}\right)=\left(k_{h 1} /(2 \omega), k_{h 2} /(2 \omega)\right)$ are the horizontal slowness vectors defined in midpoint-half-offset space, where $\left(k_{x 1}, k_{x 2}\right)$ and $\left(k_{h 1}, k_{h 2}\right)$ are the corresponding wavenumber vectors. The geometric explanation of these quantities can be seen in Figure 1.

From equation A-14 given in Appendix A, we conclude that the source slowness vector $\left(p_{s 1}, p_{s 2}\right)$ and the receiver slowness vector $\left(p_{g 1}, p_{g 2}\right)$ are linearly related to the half-offset slowness vector $\left(p_{h 1}, p_{h 2}\right)$ and the midpoint slowness components $\left(p_{x 1}, p_{x 2}\right)$

$$
\begin{aligned}
& p_{s 1}=p_{x 1}-p_{h 1}, \\
& p_{s 2}=p_{x 2}-p_{h 2}, \\
& p_{g 1}=p_{x 1}+p_{h 1}, \\
& p_{g 2}=p_{x 2}+p_{h 2} .
\end{aligned}
$$

Thus, equation 2 can be written in terms of slownesses for source and receiver:

$$
T=\left(q_{s}+q_{g}\right) z+p_{s 1} y_{s 1}+p_{s 2} y_{s 2}+p_{g 1} y_{g 1}+p_{g 2} y_{g 2},
$$

where $\left(y_{s 1}, y_{s 2}\right)$ denote projections of the lateral distance between image point and source given by $\left(y_{s 1}, y_{s 2}\right)=\left(x_{1}^{0}-h_{1}^{0}-x_{1}\right.$, $\left.x_{2}^{0}-h_{2}^{0}-x_{2}\right)$ and $\left(y_{g 1}, y_{g 2}\right)$ denotes projections of the lateral distance between image point and receiver given by $\left(y_{g 1}, y_{g 2}\right)=\left(x_{1}^{0}+\right.$ $\left.h_{1}^{0}-x_{1}, x_{2}^{0}+h_{2}^{0}-x_{2}\right)$. Equation 4 represents the total traveltime shift of the plane-wave propagations from the source located at $\left(x_{1}^{0}-h_{1}^{0}, x_{2}^{0}-h_{2}^{0}, 0\right)$ to the image point located at $\left(x_{1}, x_{2}, z\right)$ and back to the receiver located at $\left(x_{1}^{0}+h_{1}^{0}, x_{2}^{0}+h_{2}^{0}, 0\right)$.

The integrals in equation 1 can be estimated by the stationary phase method, approximately. The main contributions to the integrand come from the stationary phase in which the phase function is either the minimum or the maximum. In this case, the source and receiver slownesses at the stationary point correspond to the seismic rays that link the source, receiver, and scattering point. Hence, we can use the stationary phase method (Alkhalifah, 2000b) to derive the traveltime of the seismic ray from source to image point then back to receiver. According to equation 3, the stationary point of the phase function given in equation 1 can be found from the following equations:

$$
\frac{\partial T}{\partial p_{1(s, g)}}=0
$$

and

$$
\frac{\partial T}{\partial p_{2(s, g)}}=0
$$

where $\left(p_{1}, p_{2}\right)$ are either $\left(p_{s 1}, p_{s 2}\right)$ for the source or the receiver, respectively. Equations 5 and 6 are the mathematical representations of Fermat's principle. 
Substituting equation 4 into equations 5 and 6, respectively, we obtain the following relations:

$$
\frac{\partial q_{(s, g)}}{\partial p_{1(s, g)}}=-\frac{y_{1(s, g)}}{z}
$$

and

$$
\frac{\partial q_{(s, g)}}{\partial p_{2(s, g)}}=-\frac{y_{2(s, g)}}{z}
$$

where $q$ and $\left(y_{1}, y_{2}\right)$ are either $q_{s}$ and $\left(y_{s 1}, y_{s 2}\right)$ for source or $q_{g}$ and $\left(y_{g 1}, y_{g 2}\right)$ for receiver. Equations 4,7 , and 8 represent the exact traveltime of the seismic ray from the source to the image point then back to the receiver.

\section{SLOWNESS APPROXIMATION AT A STATIONARY POINT IN 3D TRANSVERSELY ISOTROPIC MEDIA WITH A HORIZONTAL SYMMETRY AXIS}

HTI and VTI media can be treated as special cases of the more complex orthorhombic media. In homogeneous VTI media, P- and $\mathrm{SV}$-waves can be described by the vertical velocities of P- and SVwaves and two Thomsen (1986) parameters $\varepsilon$ and $\delta$. In practice, we can ignore the influence of SV-wave vertical velocity on P-wave velocity and traveltime in TI media (Tsvankin and Thomsen, 1994; Alkhalifah, 1998; Zhou and Greenhalgh, 2008). Hence, the velocity and traveltime of P-wave in VTI media can be described by the P-wave vertical velocity, the normal-moveout velocity $v_{\text {nmo }}=$ $v_{0} \sqrt{1+2 \delta}$, and the anellipticity parameter $\eta=(\varepsilon-\delta) /(1+2 \delta)$. Besides the three parameters mentioned above, an additional parameter, the azimuth $\phi$ of the symmetry axis, is introduced to depict the azimuthal feature of the velocity and traveltime for P-waves in $3 \mathrm{D}$ HTI media.

Equations 4, 7, and 8 depend on the horizontal and vertical slowness projections. To obtain the slownesses for the source and receiver, we consider the slowness surface equation for 3D HTI media.

According to Alkhalifah (1998, 2000a), the 3D phase slowness surface for the P-wave in a VTI medium can be written in the form,

$$
\begin{aligned}
F_{\mathrm{VTI}}= & v_{0}^{2} q_{v}^{2}\left(1-2 \eta v_{\mathrm{nmo}}^{2}\left(p_{v 1}^{2}+p_{v 2}^{2}\right)\right) \\
& +(1+2 \eta) v_{\mathrm{nmo}}^{2}\left(p_{v 1}^{2}+p_{v 2}^{2}\right)-1=0,
\end{aligned}
$$

where $\left(p_{v 1}, p_{v 2}\right)$ and $q_{v}$ are the horizontal and vertical slowness components, respectively. Equation 9 is valid for smoothly heterogeneous VTI media.

The slowness surface in a 3D HTI medium can be obtained from the VTI version by a slowness rotation. In Figure 2, the $p_{v 1}, p_{v 2}$, and $q_{v}$ system denotes the slowness in VTI media; and the $p_{1}, p_{2}$, and $q$ system denotes the slowness in HTI media; $p_{v 2}$ and $q_{v}$ are located on the $p_{1}-p_{2}$ plane.

The 3D slowness surface equation for an HTI medium is obtained by applying the slowness rotation:

$$
\left(\begin{array}{c}
p_{1} \\
p_{2} \\
q
\end{array}\right)=\left(\begin{array}{ccc}
0 & -\sin \phi & \cos \phi \\
0 & \cos \phi & \sin \phi \\
-1 & 0 & 0
\end{array}\right)\left(\begin{array}{c}
p_{v 1} \\
p_{v 2} \\
q_{v}
\end{array}\right)
$$

with $\phi$ being the azimuth of the $q_{v}$-axis measured from the $p_{1}$-axis on the $p_{1}-p_{2}$ plane.

Equation 10 shows the transformation of the slowness components from VTI to HTI media. The slowness surface in an HTI medium is

$$
q=q\left(p_{1}, p_{2}\right)
$$

and the slowness surface for a VTI medium is

$$
q_{v}=q_{v}\left(p_{v 1}, p_{v 2}\right)
$$

The perturbation of equation 10 can be written in the form

$$
\left(\begin{array}{c}
\Delta p_{1} \\
\Delta p_{2} \\
\Delta q
\end{array}\right)=\left(\begin{array}{ccc}
0 & -\sin \phi & \cos \phi \\
0 & \cos \phi & \sin \phi \\
-1 & 0 & 0
\end{array}\right)\left(\begin{array}{c}
\Delta p_{v 1} \\
\Delta p_{v 2} \\
\Delta q_{v}
\end{array}\right),
$$

and the perturbation of vertical slownesses $q$ and $q_{v}$ can be obtained by differentiating equations 11 and 12 , respectively,

$$
\Delta q=\frac{\partial q}{\partial p_{1}} \Delta p_{1}+\frac{\partial q}{\partial p_{2}} \Delta p_{2}
$$

and

$$
\Delta q_{v}=\frac{\partial q_{v}}{\partial p_{v 1}} \Delta p_{v 1}+\frac{\partial q}{\partial p_{v 2}} \Delta p_{v 2}
$$

To express the partial derivatives of slowness in an HTI medium in terms of that in a VTI medium, we consider two special cases. For the first case, we assume that the perturbation of the slowness surface in a VTI medium is caused by the change in only $p_{v 1}$, not $p_{v 2}$; that is,

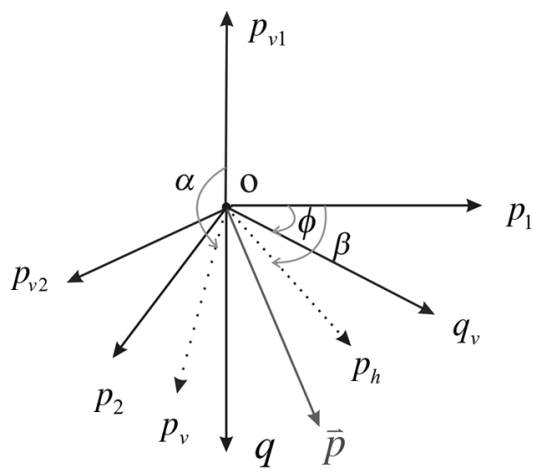

Figure 2. The geometry of the slowness coordinate system. The slowness vector $\left(p_{v 1}, p_{v 2}, q_{v}\right)$ is the projection of slowness $p$ in a VTI medium, and $\left(p_{1}, p_{2}, q\right)$ is the projection of slowness $p$ in an HTI medium. The symmetry axis of the VTI medium is along the $q_{v}$-axis, whereas $p_{v 2}$ and $q_{v}$ are located on the $p_{1}-p_{2}$ plane, and $\phi$ denotes the azimuth of $q_{v}$ measured from $p_{1}$-axis. Also, $p_{v}$ denotes the projection of slowness $\vec{p}$ in the $p_{v 1}-p_{v 2}$ plane; $\alpha$ is the azimuth of $p_{v}$ measured from the $p_{v 1}$-axis; $p_{h}$ denotes the projection of slowness $\vec{p}$ in the $p_{1}-p_{2}$ plane; and $\beta$ is the azimuth of $p_{h}$ measured from the $p_{1}$-axis. 


$$
\Delta p_{v 2}=0 .
$$

Hence, from equations 13-15, we obtain the following linear equation:

$$
\left(\begin{array}{ccc}
-1 & 0 & \cos \phi \frac{\partial q_{v}}{\partial p_{v 1}} \\
0 & -1 & \sin \phi \frac{\partial q_{v}}{\partial p_{v 1}} \\
\frac{\partial q}{\partial p_{1}} & \frac{\partial q}{\partial p_{2}} & 1
\end{array}\right)\left(\begin{array}{c}
\Delta p_{1} \\
\Delta p_{2} \\
\Delta p_{v 1}
\end{array}\right)=0 .
$$

The existence of a nonzero solution of equation 17 implies that the determinant of the coefficient matrix given in equation 17 is zero. Hence, we obtain the expression for $\partial q_{v} / \partial p_{v 1}$,

$$
\frac{\partial q_{v}}{\partial p_{v 1}}=-\left(\cos \phi \frac{\partial q}{\partial p_{1}}+\sin \phi \frac{\partial q}{\partial p_{2}}\right)^{-1} .
$$

For the second case, we assume that the perturbations of slowness surface in a VTI medium is caused by the change in only $p_{v 2}$ not $p_{v 1}$; that is,

$$
\Delta p_{v 1}=0
$$

A similar procedure can be performed for equations 13-15. Consequently, we obtain the expression

$$
\frac{\partial q_{v}}{\partial p_{v 2}}=-\frac{\cos \phi \frac{\partial q}{\partial p_{2}}-\sin \phi \frac{\partial q}{\partial p_{1}}}{\cos \phi \frac{\partial q}{\partial p_{1}}+\sin \phi \frac{\partial q}{\partial p_{2}}} .
$$

Equations 18 and 20 describe the partial derivative relations between VTI and HTI media. A similar idea is used for mapping of moveout functions in TI media (Stovas and Alkhalifah, 2014). By substituting equations 7 and 8 into equations 18 and 20, we represent the slowness derivatives in terms of spatial coordinates in an HTI medium:

$$
\frac{\partial q_{v}}{\partial p_{v 1}}=\frac{z}{y_{1} \cos \phi+y_{2} \sin \phi}
$$

and

$$
\frac{\partial q_{v}}{\partial p_{v 2}}=-\frac{y_{2} \cos \phi-y_{1} \sin \phi}{y_{1} \cos \phi+y_{2} \sin \phi}
$$

To derive the expressions for $p_{v 1}$ and $p_{v 2}$ from equations 21 and 22 , we introduce the radial horizontal slowness $p_{v}$ and the azimuth $\alpha$ (see Figure 2) using the following relations:

$$
p_{v 1}=p_{v} \cos \alpha
$$

and

$$
p_{v 2}=p_{v} \sin \alpha .
$$

Because the vertical slowness component $q_{v}$ in a VTI medium is the function of the horizontal slowness component $p_{v}$, it follows that the first-order derivatives $\partial q_{v} / \partial p_{v 1}$ and $\partial q_{v} / \partial p_{v 2}$ are given by

$$
\frac{\partial q_{v}}{\partial p_{v 1}}=\frac{d q_{v}}{d p_{v}} \cos \alpha
$$

and

$$
\frac{\partial q_{v}}{\partial p_{v 2}}=\frac{d q_{v}}{d p_{v}} \sin \alpha
$$

From equations 21, 22, 25, and 26, we obtain the expressions for $d q_{v} / d p_{v}$ and $\tan \alpha$ :

$$
\begin{aligned}
\frac{d q_{v}}{d p_{v}} & =\sqrt{\left(\frac{\partial q_{v}}{\partial p_{v 1}}\right)^{2}+\left(\frac{\partial q_{v}}{\partial p_{v 2}}\right)^{2}} \\
& =\frac{\sqrt{\left(y_{2} \cos \phi-y_{1} \sin \phi\right)^{2}+z^{2}}}{\left|y_{1} \cos \phi+y_{2} \sin \phi\right|} \\
& =c
\end{aligned}
$$

and

$$
\tan \alpha=\frac{\partial q_{v}}{\partial p_{v 2}} / \frac{\partial q_{v}}{\partial p_{v 1}}=-\left(\frac{y_{2}}{z} \cos \phi-\frac{y_{1}}{z} \sin \phi\right) .
$$

From equations 9, 23, and 24, we can obtain the explicit expression for $q_{v}$ in terms of $p_{v}$. Then, substituting it into equation 27, we obtain the equation for the horizontal slowness component $p_{v}$,

$$
p_{v}^{2} v_{\text {nmo }}^{4}-c^{2} v_{0}^{2}\left(-1+2 p_{v}^{2} v_{\text {nmo }}^{2} \eta\right)^{3}\left(-1+p_{v}^{2} v_{\text {nmo }}^{2}(1+2 \eta)\right)=0 .
$$

From equations 9, 23, and 24, we can also obtain $p_{v}$ represented in terms of $q_{v}$. Similarly, we derive the equation for the vertical slowness component $q_{v}$ given by

$$
c^{2} q_{v}^{2} v_{0}^{4}-\left(1-q_{v}^{2} v_{0}^{2}\right) v_{\text {nmo }}^{2}\left(1+2 \eta-2 q_{v}^{2} v_{0}^{2} \eta\right)^{3}=0 .
$$

Equations 29 and 30 are quartic equations with respect to variables $p_{v}^{2}$ and $q_{v}^{2}$, respectively. Under the assumption of weak anellipticity of HTI media, we use the second-order perturbation of $p_{v}$ and $q_{v}$ in the anellipticity parameter $\eta$ (considering that it is small) to obtain the following approximations:

$$
p_{v}=-r\left[p_{v 0}+p_{v 1}(2 \eta)+p_{v 2}(2 \eta)^{2}\right]
$$

and

$$
q_{v}=r\left[q_{v 0}+q_{v 1}(2 \eta)+q_{v 2}(2 \eta)^{2}\right],
$$

where we imply that the sums in the square brackets are positive and the function $r$ controls the sign selection for $p_{v}$ and $q_{v}$ :

$$
r=\frac{y_{1} \cos \phi+y_{2} \sin \phi}{\left|y_{1} \cos \phi+y_{2} \sin \phi\right|} \text {. }
$$

The selection of signs for $p_{v}$ and $q_{v}$ are given in Appendix B. 
By substituting equation 31 into equation 29, we obtain a polynomial equation in $\eta$. Because equation 31 is the trial solution of equation 29, it follows that all coefficients in this polynomial equation should be zero. Consequently, we derive

$$
\begin{gathered}
p_{v 0}=\frac{c v_{0}}{v_{\mathrm{nmo}} \sqrt{c^{2} v_{0}^{2}+v_{\mathrm{nmo}}^{2}}}, \\
p_{v 1}=-\frac{c^{3} v_{0}^{3}\left(c^{2} v_{0}^{2}+4 v_{\mathrm{nmo}}^{2}\right)}{2 v_{\mathrm{nmo}}\left(c^{2} v_{0}^{2}+v_{\mathrm{nmo}}^{2}\right)^{5 / 2}},
\end{gathered}
$$

and

$$
p_{v 2}=\frac{3 c^{5} v_{0}^{5}\left(c^{4} v_{0}^{4}+4 c^{2} v_{0}^{2} v_{\mathrm{nmo}}^{2}+24 v_{\mathrm{nmo}}^{4}\right)}{8 v_{\mathrm{nmo}}\left(c^{2} v_{0}^{2}+v_{\mathrm{nmo}}^{2}\right)^{9 / 2}}
$$

In a similar way, we obtain coefficients $q_{v i}, i=0,1,2$,

$$
\begin{aligned}
& q_{v 0}=\frac{v_{\mathrm{nmo}}}{v_{0} \sqrt{c^{2} v_{0}^{2}+v_{\mathrm{nmo}}^{2}}}, \\
& q_{v 1}=\frac{3 c^{4} v_{0}^{3} v_{\mathrm{nmo}}}{2\left(c^{2} v_{0}^{2}+v_{\mathrm{nmo}}^{2}\right)^{5 / 2}},
\end{aligned}
$$

and

$$
q_{v 2}=\frac{3 c^{6} v_{0}^{5} v_{\mathrm{nmo}}\left(c^{2} v_{0}^{2}-20 v_{\mathrm{nmo}}^{2}\right)}{8\left(c^{2} v_{0}^{2}+v_{\mathrm{nmo}}^{2}\right)^{9 / 2}}
$$

Shanks transformation (Bender and Orszag, 1978, pp. 369-375) is used to improve the accuracy of the trial solutions for the horizontal and vertical slowness components $p_{v}$ and $q_{v}$ in a VTI medium. The final results become

$$
p_{v}=-r\left(p_{v 0}+\frac{2 p_{v 1}^{2} \eta}{p_{v 1}-2 p_{v 2} \eta}\right)
$$

and

$$
q_{v}=r\left(q_{v 0}+\frac{2 q_{v 1}^{2} \eta}{q_{v 1}-2 q_{v 2} \eta}\right)
$$

where the expression for $r$ is given by equation 33. The derivations of equations 40 and 41 can be seen in Appendix C.

From equations 10,23 , and 24 , we obtain the norm $p$ of the horizontal slowness vector, the azimuth $\beta$ of the horizontal slowness vector measured from $x$-axis (see Figure 2), and the vertical slowness component in a 3D HTI medium,

$$
\begin{gathered}
p=\sqrt{p_{1}^{2}+p_{2}^{2}}=\sqrt{p_{v}^{2} \sin ^{2} \alpha+q_{v}^{2}}, \\
\tan \beta=p_{2} / p_{1}=\frac{p_{v} \cos \phi \sin \alpha+q_{v} \sin \phi}{-p_{v} \sin \phi \sin \alpha+q_{v} \cos \phi},
\end{gathered}
$$

and

$$
q=-p_{v} \cos \alpha
$$

where the azimuth $\alpha$ is defined in equation 28 .

Equations 42-44 show that the P-wave slowness $\left(p_{1}, p_{2}, q\right)$ in an HTI medium at the stationary point can be obtained by calculating the corresponding slowness in a VTI medium.

\section{AZIMUTH-DEPENDENT TIME- AND DEPTH- DOMAIN TRAVELTIME PYRAMIDS FOR 3D TRANSVERSELY ISOTROPIC MEDIA WITH A HORIZONTAL SYMMETRY AXIS}

To derive an azimuth-dependent traveltime pyramid equation, we introduce the azimuth angle $\gamma$, which denotes the observation azimuth measured from the $x$-axis (see Figure 1):

$$
\tan \gamma_{(s, g)}=y_{2(s, g)} / y_{1(s, g)},
$$

and the norm $y$ of the lateral distance projection for source and receiver, respectively,

$$
y_{(s, g)}=\sqrt{\left(y_{1(s, g)}\right)^{2}+\left(y_{2(s, g)}\right)^{2}} \text {, }
$$

where $\gamma, y$, and $\left(y_{1}, y_{2}\right)$ are either $\gamma_{s}, y_{s}$, and $\left(y_{s 1}, \mathrm{y}_{s 2}\right)$ for the source or $\gamma_{g}, y_{g}$, and $\left(y_{g 1}, \mathrm{y}_{g_{2}}\right)$ for the receiver. The expressions for $\left(y_{s 1}, \mathrm{y}_{s 2}\right)$ and $\left(y_{g 1}, \mathrm{y}_{g 2}\right)$ are given below equation 4.

Considering equations $42,43,45$, and 46 , traveltime equation 4 becomes

$$
\begin{aligned}
& T\left(x_{1}, x_{2}, x_{1}^{0}, x_{2}^{0}, h_{1}^{0}, h_{2}^{0}, z\right)=\left(q_{s}+q_{g}\right) z \\
& \quad+p_{s} y_{s} \cos \left(\gamma_{s}-\beta_{s}\right)+p_{g} y_{g} \cos \left(\gamma_{g}-\beta_{g}\right),
\end{aligned}
$$

which is the depth-domain version of the traveltime pyramid for a 3D HTI medium.

The time-domain traveltime pyramid can be derived by relating the depth $z$ to the zero-offset two-way traveltime $\tau$. Setting half-offset $\left(h_{1}^{0}, h_{2}^{0}\right)=0$ and lateral projection of image point equal to lateral midpoint projection, $\left(x_{1}, x_{2}\right)=\left(x_{1}^{0}, x_{2}^{0}\right)$ in equation 2 , we obtain the zero-offset two-way traveltime $\tau$,

$$
\tau=T\left(x_{1}, x_{2}, x_{1}^{0}=x_{1}, x_{2}^{0}=x_{2}, h_{1}^{0}=0, h_{2}^{0}=0, z\right)=2 q_{z 0} z,
$$

and the corresponding P-wave vertical slowness component $q_{z 0}$,

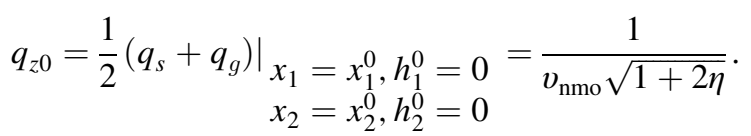

Substituting equation 48 into equation 47 , we obtain the timedomain traveltime pyramid for an HTI medium, 


$$
\begin{aligned}
& T\left(x_{1}, x_{2}, x_{1}^{0}, x_{2}^{0}, h_{1}^{0}, h_{2}^{0}, \tau\right)=\frac{v_{\mathrm{nmo}} \sqrt{1+2 \eta}}{2}\left(q_{s}+q_{g}\right) \tau \\
& \quad+p_{s} y_{s} \cos \left(\gamma_{s}-\beta_{s}\right)+p_{g} y_{g} \cos \left(\gamma_{g}-\beta_{g}\right) .
\end{aligned}
$$

For a homogeneous 3D HTI medium, we have model parameters $v_{0}, v_{\text {nmo }}, \eta$, and $\phi$. For a single image point in this medium, we can use equation 50 to analytically calculate the P-wave diffraction traveltime. In equation 50, slowness projections for source and receivers $\left(p_{s}, q_{s}\right)$ and $\left(p_{g}, q_{g}\right)$ can be analytically calculated from equations 27, 28, 33-44; azimuth angles $\left(\beta_{s}, \gamma_{s}\right)$ for source and $\left(\beta_{g}\right.$, $\gamma_{g}$ ) for receiver can be obtained from equations 43 and 45 .

Alkhalifah (2013) derives an approximate solution of the P-wave eikonal equation in inhomogeneous 3D HTI media. He proposes to expand the traveltime with respect to the azimuth of the symmetry axis $\phi$ and the anellipticity parameter $\eta$. In our derivations of the traveltime pyramid, we expand the slowness components with respect to only the anellipticity parameter $\eta$. Hence, the approximation proposed by us has the same accuracy as Alkhalifah's approximation, regardless of the expansion of traveltime with respect to azimuth $\phi$ in his derivation.

\section{AZIMUTH-DEPENDENT TRAVELTIME APPROXIMATION FOR A HORIZONTAL REFLECTOR IN 3D TRANSVERSELY ISOTROPIC MEDIA WITH A HORIZONTAL SYMMETRY AXIS}

In the case that the image point is located vertically under the midpoint, we have $\left(x_{1}-x_{1}^{0}, x_{2}-x_{2}^{0}\right)=0$ and $\left(p_{x 1}, p_{x 2}\right)=0$. Thus, equation 50 yields the traveltime equation for reflections in a horizontal reflector: a)

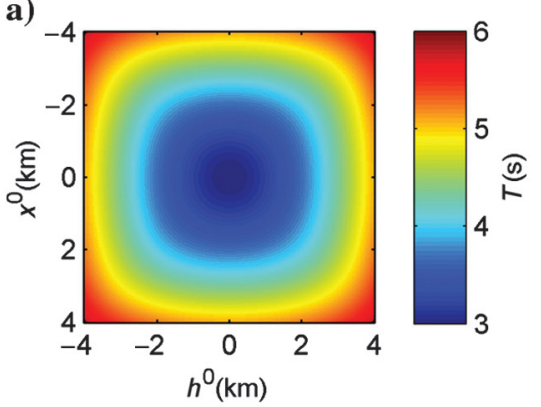

c)

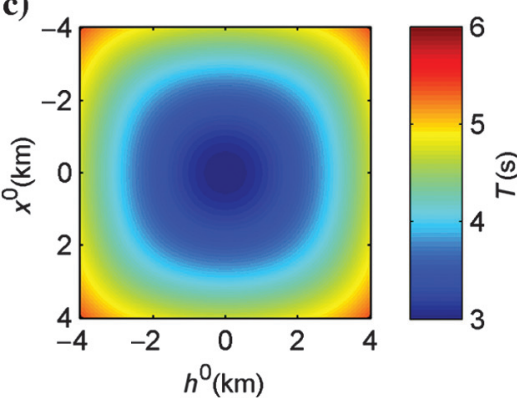

b)

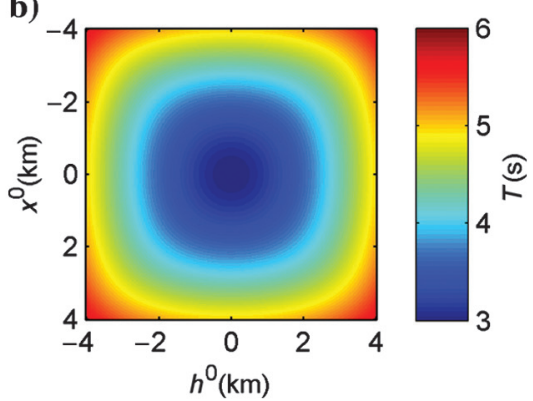

d)

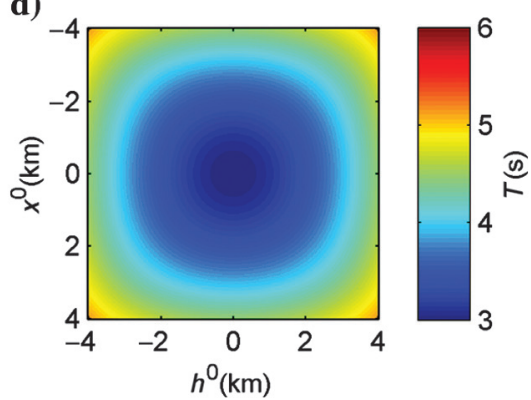

Figure 3. Traveltime as a function of the half-offset $h^{0}$ and the midpoint $x^{0}$ in a 3D HTI medium with different acquisition azimuths: (a) $\gamma=0$, (b) $\gamma=\pi / 6$, (c) $\gamma=\pi / 3$, and (d) $\gamma=\pi / 2$.

$$
T\left(h^{0}, \gamma\right)=\sqrt{1+2 \eta} v_{\mathrm{nmo}} \tau q+2 p_{h} h^{0} \cos (\gamma-\beta),
$$

where $h^{0}=\sqrt{\left(h_{1}^{0}\right)^{2}+\left(h_{2}^{0}\right)^{2}}$ denotes the norm of the half-offset projection and $\gamma-\beta$ denotes the variation between the acquisition azimuth and the horizontal slowness vector. For a homogeneous isotropic medium, $\gamma-\beta$ equals zero. In the case of an elliptical HTI medium $(\eta=0)$, we can obtain the exact expressions for $p_{h}, q$, and $\beta$ from equations $31-45$. Finally, equation 51 is reduced to

$$
T\left(h^{0}, \gamma\right)=\sqrt{\tau^{2}+4\left(h^{0}\right)^{2}\left(\frac{\sin ^{2}(\gamma-\phi)}{v_{\mathrm{nmo}}^{2}}+\frac{\cos ^{2}(\gamma-\phi)}{v_{0}^{2}}\right)} .
$$

The derivation of equation 52 is shown in Appendix D. Further simplification for the isotropic case results in the well-known hyperbolic traveltime equation:

$$
T\left(h^{0}, \gamma\right)=\sqrt{\tau^{2}+\frac{4\left(h^{0}\right)^{2}}{v_{\mathrm{nmo}}^{2}}} .
$$

\section{NUMERICAL EXAMPLES}

To examine the shape of the traveltime pyramid as a function of the half-offset and the midpoint, we design a homogeneous 3D HTI model with a symmetry axis aligned to the $x$-axis. The medium parameters are $v_{0}=2000 \mathrm{~m} / \mathrm{s}, \delta=0.1$, and $\eta=0.1$. The image point is located on the $z$-axis. The midpoint is located in the origin of the acquisition coordinate system. The zero-offset two-way traveltime $\tau=3$ s for midpoint $x^{0}=0$; from which we calculate the depth of the image point $z=\tau v_{0} \sqrt{(1+2 \delta)(1+2 \eta)} / 2=3.6 \mathrm{~km}$.

We use equation 50 to calculate traveltime pyramids. Figure 3 shows the traveltime pyramids computed for different acquisition azimuths. Only the azimuthal variation from 0 to $\pi / 2$ is considered because the traveltime is symmetric with respect to the $y$-axis. The peak of the traveltime pyramid is located at the zero-midpoint $\left(x^{0}=0\right)$ and the zero-offset $\left(h^{0}=0\right)$ for all azimuths. The traveltime function is symmetric with respect to the half-offset and the midpoint due to the horizontal symmetry of HTI media. Figure 3 shows that the acquisition azimuth $\gamma=0$ corresponds to the symmetry plane of the HTI model. With an increase in $\gamma$, the acquisition line moves away from the symmetry plane. When $\gamma=\pi / 2$, the acquisition line is located in the isotropic plane of the HTI model. It can also be seen that the shape of the traveltime pyramid varies with the change in acquisition azimuth. This behavior becomes more dramatic for large values of midpoint and offset.

Figure 4 shows slices of the traveltime pyramids from Figure 3 extracted for $x^{0}=0$ (the CMP case), $h^{0}=0$ (the common-offset case), 
a)

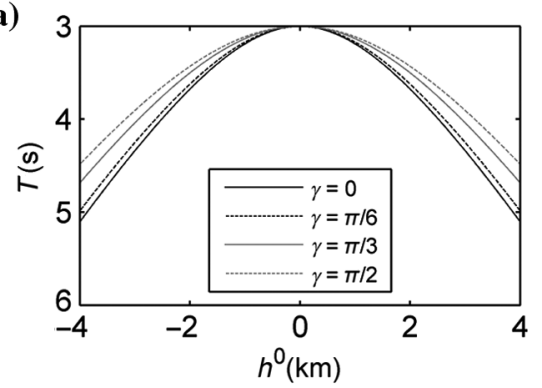

b)

c)

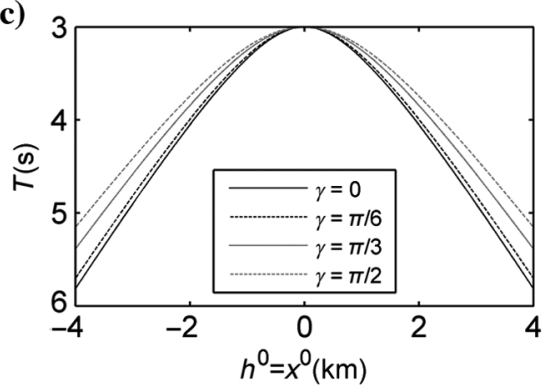

Figure 4. Comparison of azimuth-dependent traveltimes extracted from traveltime pyramids for (a) $x^{0}=0$, (b) $h^{0}=0$, and (c) $x^{0}=h^{0}$ in Figure 3.

a)

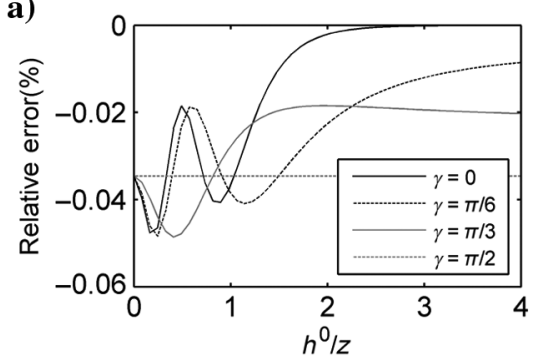

c)

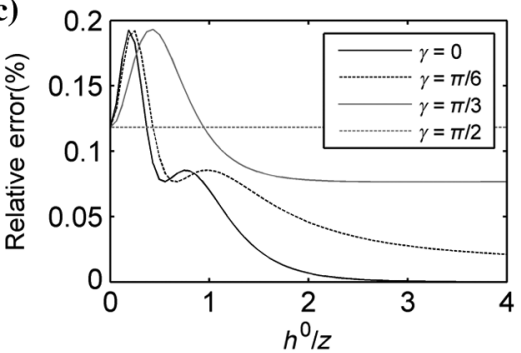

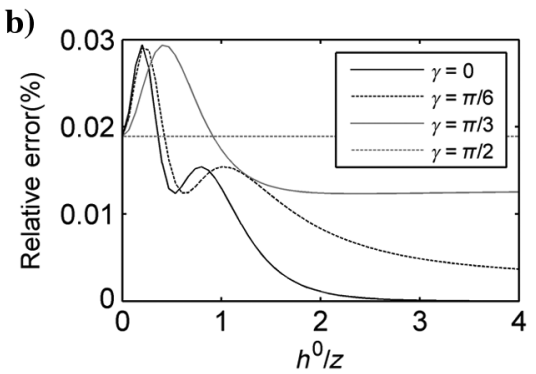

d)

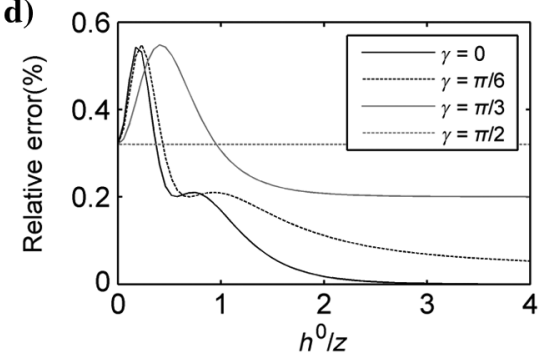

Figure 5. Relative error of azimuth-dependent traveltime equation as a function of the half-offset-to-depth ratio in 3D HTI media with $\eta$ equal to (a) -0.1 , (b) 0.1 , (c) 0.2 , and (d) 0.3 , respectively.

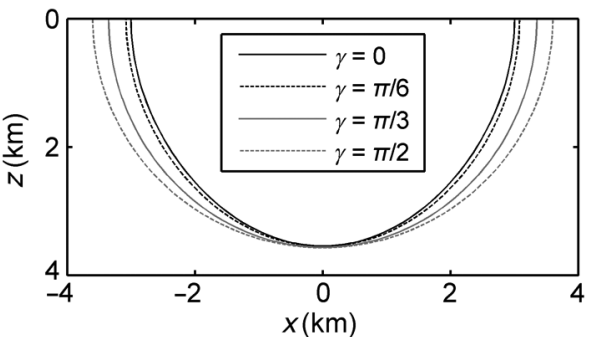

Figure 6. The common-offset migration isochrones with different acquisition azimuths. and the case of $x^{0}=h^{0}$. We can see that the traveltime decreases as the azimuth increases, and the traveltime pyramid becomes much flatter. This effect becomes more pronounced for large values of offset and midpoint.

Next, we test the accuracy of equation 51. Two-point ray tracing (Shearer and Chapman, 1988) is used to calculate the exact P-wave reflection traveltime for a horizontal reflector in a 3D HTI medium. The model parameters, except the anellipticity parameter $\eta$, are the same as in the first test. The zero-offset two-way traveltime is $\tau=3 \mathrm{~s}$. Figure 5 compares the relative errors in traveltime for HTI models with different $\eta$. The result for $\eta=0$ is not shown here because in this case our approximation equation 51 is reduced to the exact traveltime equation 52 for the 3D elliptical HTI model. We can see that the traveltime approximation 51 is very accurate for all azimuths with a maximum relative error of $0.05 \%$ in HTI models with weak anellipticity $(\eta=-0.1$ and $\eta=0.1)$. The increase of error versus $\eta$ is significant at a short offset $\left(h^{0} / z \leq 1\right)$ due to the weak anellipticity assumption in our derivations of equations 50 and 51 . The zero-offset traveltime is not exact because the vertical slowness in a 3D HTI medium is an approximate value due to the Taylor series expansion in $\eta$.

We also show the variation of common-offset migration isochrones with the change in acquisition azimuth. We use equation 50 to calculate the migration isochrones. The model parameters from the first example are adopted. To obtain the common-offset isochrones, we set the half-offset $h^{0}$ and traveltime $t$ to be constants, $h^{0}=500 \mathrm{~m}$ and $t=3 \mathrm{~s}$ regardless to the azimuths. Figure 6 shows the migration isochrones corresponding to azimuth $\gamma=0, \pi / 6, \pi / 3, \pi / 2$. Azimuth $\gamma=\pi / 2$ corresponds to the isotropic plane, and azimuth $\gamma=0$ corresponds to the symmetry plane of the HTI medium. The azimuthal variations of migration isochrones are more significant at large dip angles. This indicates that the ignorance of the azimuthal anisotropy can produce significant migration errors in HTI media.

\section{CONCLUSIONS}

The azimuth-dependent offset-midpoint traveltime pyramid for homogeneous 3D HTI media is derived under the assumption of weak anellipticity. Perturbation in anellipticity parameter $\eta$ and using the Shanks transformation help obtain a relatively simple analytic form for the traveltime approximation. The azimuth-offset traveltime equation for reflected waves in a horizontal 3D HTI reflector is obtained from the traveltime pyramid. Despite the approximations, the equations are reasonably accurate and can be used for imaging and parameter estimation applications.

The derivation of traveltime pyramids can be extended to the case of a 3D homogeneous TTI medium and be used to derive 
the traveltime approximation of reflected wave in a 3D dipconstrained transversely isotropic model.

\section{ACKNOWLEDGMENTS}

We would like to acknowledge the rock and seismic (ROSE) project for financial support. We thank the King Abdullah University for Science and Technology for its financial support of this work. We also acknowledge assistant editor V. Socco, associate editor V. Vinje, B. Zhou, X. Song, and two anonymous reviewers for many valuable remarks.

\section{APPENDIX A \\ PRESTACK PHASE-SHIFT MIGRATION OPERATOR IN MIDPOINT-HALF-OFFSET DOMAIN}

Here, we derive the midpoint-half-offset domain prestack phaseshift migration operator for a $\mathrm{P}$-wave in homogeneous anisotropic media. In advance of our following derivations, we refer to the Fourier transform convention shown in Yilmaz (2001, p. 156). The 2D Fourier transform of a time-space domain wavefield $P(x, t)$ is given by

$$
P(k, \omega)=\iint P(x, t) \exp (i k x-i \omega t) d x d t
$$

Wavefield $P(x, t)$ can be reconstructed from $P(k, \omega)$ by the $2 \mathrm{D}$ inverse Fourier transform:

$$
P(x, t)=\iint P(k, \omega) \exp (-i k x+i \omega t) d k d \omega .
$$

The dispersion relation for P-wave in general anisotropic media can be represented by

$$
S\left(k_{1}, k_{2}, k_{z}, \mathbf{v}\right)=\omega^{2},
$$

where $\left(k_{1}, k_{2}\right)$ denote the horizontal wavenumber vector; $k_{z}$ denotes the vertical wavenumber component; $\mathbf{v}$ denotes the vector containing the medium parameters; and $S$ denotes the dispersion function of $k_{1}, k_{2}, k_{z}$, and $\mathbf{v}$, which can be explicitly obtained for a specified anisotropic medium (Carcione, 2001, pp. 10-11). Solving equation A-3 for $k_{z}^{2}$ gives

$$
k_{z}^{2}=k_{z}^{2}\left(k_{1}, k_{2}, \omega, \mathbf{v}\right) .
$$

Transforming equation A-4 from the vertical wavenumber domain back to the depth domain gives the depth-dependent pseudoacoustic equation:

$$
\frac{\partial^{2}}{\partial z^{2}} P\left(k_{1}, k_{2}, z, \omega\right)+k_{z}^{2}\left(k_{1}, k_{2}, \omega, \mathbf{v}(z)\right) P\left(k_{1}, k_{2}, z, \omega\right)=0 .
$$

For a homogeneous medium, the medium parameter vector $\mathbf{v}(z)$ becomes depth independent. In this case, we derive the depth domain wavefield extrapolation for the one-way $\mathrm{P}$-wave given by

$$
\begin{aligned}
P\left(k_{1}, k_{2}, z, \omega\right)= & P\left(k_{1}, k_{2}, z=0, \omega\right) \\
& \times \exp \left[+i z \sqrt{k_{z}^{2}\left(k_{1}, k_{2}, \omega, \mathbf{v}\right)}\right] .
\end{aligned}
$$

Here, the choice of a plus sign "+" in the square brackets means that $\exp \left(i \omega t+i k_{z} z\right)$ is an upcoming wave according to the convention of inverse Fourier transform defined in equation A-2.

Equation A-6 illustrates that a 3D prestack wavefield for the upcoming P-wave recorded at the earth's surface can be extrapolated downward to depth $z$. For a prestack 3D wavefield in the sourcereceiver domain, the vertical wavenumber component is expressed as a sum of two square roots: One is associated with downward continuation of shots and another one is associated with downward continuation of receivers (Yilmaz, 2001, pp. 631-632):

$$
\begin{aligned}
k_{z} & =k_{s z}+k_{g z} \\
& =\sqrt{k_{z}^{2}\left(k_{s 1}, k_{s 2}, \omega, \mathbf{v}\right)}+\sqrt{k_{z}^{2}\left(k_{g 1}, k_{g 2}, \omega, \mathbf{v}\right)},
\end{aligned}
$$

where $\left(k_{s 1}, k_{s 2}\right)$ and $\left(k_{g 1}, k_{g 2}\right)$ denote the horizontal slowness vectors for the source and receiver, respectively; $k_{s z}$ and $k_{g z}$ denote the vertical wavenumber components for source and receiver. Hence, we obtain the depth domain wavefield extrapolation for the twoway $\mathrm{P}$-wave in source-receiver domain given by

$$
\begin{aligned}
P\left(k_{s 1}, k_{s 2}, k_{g 1}, k_{g 2}, z, \omega\right)= & P\left(k_{s 1}, k_{s 2}, k_{g 1}, k_{g 2}, z=0, \omega\right) \\
& \times \exp \left(i k_{z} z\right),
\end{aligned}
$$

where the vertical wavenumber component $k_{z}$ is given by equation A-7. Corresponding to the frequency-wavenumber domain wavefield $P\left(k_{s 1}, k_{s 2}, k_{g 1}, k_{g 2}, z, \omega\right)$, the time-space domain wavefield $P\left(s_{1}, s_{2}, g_{1}, g_{2}, z, t\right)$ is obtained by the inverse Fourier transform:

$$
\begin{aligned}
& P\left(s_{1}, s_{2}, g_{1}, g_{2}, z, t\right) \\
& \quad=\iiint \iint P\left(k_{s 1}, k_{s 2}, k_{g 1}, k_{g 2}, z, \omega\right) \\
& \quad \times \exp (-i \Psi) d \omega d k_{s 1} d k_{s 2} d k_{g 1} d k_{g 2},
\end{aligned}
$$

where the phase shift $\Psi$ is given by

$$
\Psi=k_{s 1} s_{1}+k_{s 2} s_{2}+k_{g 1} g_{1}+k_{g 2} g_{2}-\omega t
$$

Substitution of equation A-8 into equation A-9 results in the prestack source-receiver domain wavefield extrapolation:

$$
\begin{aligned}
& P\left(s_{1}, s_{2}, g_{1}, g_{2}, z, t\right)=\iiint \int P\left(k_{s 1}, k_{s 2}, k_{g 1}, k_{g 2}, z=0, \omega\right) \\
& \times \exp \left(-i \Phi_{z}\right) d \omega d k_{s 1} d k_{s 2} d k_{g 1} d k_{g 2},
\end{aligned}
$$

where

$$
\Phi=-k_{z}+k_{s 1} \frac{s_{1}}{z}+k_{s 2} \frac{s_{2}}{z}+k_{g 1} \frac{g_{1}}{z}+k_{g 2} \frac{g_{2}}{z}-\omega \frac{t}{z} .
$$

Here, the expression for $k_{z}$ is given in equation A-7. 
From the geometric relation between the shot-receiver and midpoint-half-offset acquisition systems, we obtain

$$
\begin{aligned}
& s_{1}=x_{1}-h_{1}, \\
& s_{2}=x_{2}-h_{2}, \\
& g_{1}=x_{1}+h_{1},
\end{aligned}
$$

and

$$
g_{2}=x_{2}+h_{2} \text {, }
$$

where $\left(x_{1}, x_{2}\right)$ and $\left(h_{1}, h_{2}\right)$ denote the midpoint and half-offset vectors, respectively. It follows that the corresponding wavenumber relations are (Claerbout, 1985, p. 181)

$$
\begin{aligned}
& k_{s 1}=\left(k_{x 1}-k_{h 1}\right) / 2, \\
& k_{s 2}=\left(k_{x 2}-k_{h 2}\right) / 2, \\
& k_{g 1}=\left(k_{x 1}+k_{h 1}\right) / 2,
\end{aligned}
$$

and

$$
k_{g 2}=\left(k_{x 2}+k_{h 2}\right) / 2,
$$

where $\left(k_{x 1}, k_{x 2}\right)=\left(2 \omega p_{x 1}, 2 \omega p_{x 2}\right)$ and $\left(k_{h 1}, k_{h 2}\right)=\left(2 \omega p_{h 1}, 2 \omega p_{h 2}\right)$ denote the horizontal slowness vectors for the midpoint and half-offset (Alkhalifah, 2000b) and $\left(p_{x 1}, p_{x 2}\right)$ and $\left(p_{h 1}, p_{h 2}\right)$ are the corresponding slowness vectors for the midpoint and half-offset. Through the wavenumber transform operator A-14, we can convert the sourcereceiver domain wavefield to the midpoint-half-offset domain wavefield. Similar to the source-receiver domain wavefield extrapolation mentioned above, the midpoint-half-offset domain wavefield extrapolation is written as

$$
\begin{aligned}
P\left(x_{1}, x_{2}, h_{1}, h_{2}, z, t\right) & =\iiint \iint P\left(k_{x 1}, k_{x 2}, k_{h 1}, k_{h 2}, z=0, \omega\right) \\
& \times \exp (-i \Theta z) d \omega d k_{x 1} d k_{x 2} d k_{h 1} d k_{h 2},
\end{aligned}
$$

where $P\left(x_{1}, x_{2}, h_{1}, h_{2}, z, t\right)$ denotes the extrapolated CMP gathers at depth $z ; P\left(k_{x 1}, k_{x 2}, k_{h 1}, k_{h 2}, z=0, \omega\right)$ denotes the wavefield in frequency-wavenumber domain at the surface; and the phase-shift $\Theta$ is given by

$$
\begin{aligned}
\Theta & =-\operatorname{DSR}\left(k_{x 1}, k_{x 2}, k_{h 1}, k_{h 2}\right)+k_{x 1} \frac{x_{1}}{z} \\
& +k_{x 2} \frac{x_{2}}{z}+k_{h 1} \frac{h_{1}}{z}+k_{g 2} \frac{h_{2}}{z}-\omega \frac{t}{z},
\end{aligned}
$$

where $\operatorname{DSR}\left(k_{x 1}, k_{x 2}, k_{h 1}, k_{h 2}\right)$ denotes the DSR operator derived from equation A-7:

$$
\begin{aligned}
\operatorname{DSR}\left(x_{1}, x_{2}, h_{1}, h_{2}\right) & =\sqrt{k_{z}^{2}\left(\left(k_{x 1}-k_{h 1}\right) / 2,\left(k_{x 2}-k_{h 2}\right) / 2, \omega, \mathbf{v}\right)} \\
& +\sqrt{k_{z}^{2}\left(\left(k_{x 1}+k_{h 1}\right) / 2,\left(k_{x 2}+k_{h 2}\right) / 2, \omega, \mathbf{v}\right)} \\
& =k_{s z}+k_{g z} .
\end{aligned}
$$

By setting $t=0$ and $\left(h_{1}, h_{2}\right)=(0,0)$ in equation A- 15 , we obtain the prestack phase-shift migration operator in the midpoint-half-offset domain:

$$
\begin{aligned}
& P\left(x_{1}, x_{2}, h_{1}=0, h_{2}=0, z, t=0\right) \\
& \quad=\iiint \iint P\left(k_{x 1}, k_{x 2}, k_{h 1}, k_{h 2}, z=0, \omega\right) \\
& \quad \times \exp (-i \Theta z) d \omega d k_{x 1} d k_{x 2} d k_{h 1} d k_{h 2},
\end{aligned}
$$

where $\Theta$ becomes

$$
\Theta=-\operatorname{DSR}\left(x_{1}, x_{2}, h_{1}, h_{2}\right)+k_{x 1} \frac{x_{1}}{z}+k_{x 2} \frac{x_{2}}{z} .
$$

To obtain the response of applying the prestack phase-shift migration A-18 on a single trace, we adopt the following definition (Alkhalifah, 2000b):

$$
\begin{aligned}
& P\left(x_{1}, x_{2}, h_{1}, h_{2}, z=0, t\right) \\
& \quad=\tilde{P}\left(x_{1}, x_{2}, h_{1}, h_{2}, z=0, t\right) \delta\left(x_{1}-x_{1}^{0}, x_{2}-x_{2}^{0}, h_{1}-h_{1}^{0}, h_{2}-h_{2}^{0}\right),
\end{aligned}
$$

where $\tilde{P}\left(x_{1}, x_{2}, h_{1}, h_{2}, z=0, t\right)$ denotes the time-space domain seismic record at surface and $\delta(\ldots)$ denotes the Dirac delta function. The midpoint position $\left(x_{1}^{0}, x_{2}^{0}\right)$ and the half-offset $\left(h_{1}^{0}, h_{2}^{0}\right)$ specify the spatial position of the single trace. The Fourier transform of the time-space domain wavefield $P\left(x_{1}, x_{2}, h_{1}, h_{2}, z, t\right)$ is given by

$$
\begin{aligned}
& P\left(k_{x 1}, k_{x 2}, k_{h 1}, k_{h 2}, z, \omega\right) \\
& =\iiint \iint P\left(x_{1}, x_{2}, h_{1}, h_{2}, z, t\right) \\
& \times \exp (i \Upsilon) d t d x_{1} d x_{2} d h_{1} d h_{2},
\end{aligned}
$$

where phase shift $r$ is

$$
r=k_{x 1} x_{1}+k_{x 2} x_{2}+k_{h 1} h_{1}+k_{h 2} h_{2}-\omega t .
$$

From equations A-20-A-22, we derive the wavefield in frequencywavenumber domain:

$$
\begin{aligned}
& P\left(k_{x 1}, k_{x 2}, k_{h 1}, k_{h 2}, z=0, \omega\right) \\
& \quad=\tilde{P}\left(x_{1}^{0}, x_{2}^{0}, h_{1}^{0}, h_{2}^{0}, z=0, \omega\right) \\
& \quad \times \exp \left(i k_{x 1} x_{1}^{0}+i k_{x 2} x_{2}^{0}+i k_{h 1} h_{1}^{0}+i k_{h 2} h_{2}^{0}\right) .
\end{aligned}
$$

By substituting equation A-23 into equation A-18, we obtain the single-trace response of the $3 \mathrm{D}$ prestack phase-shift migration in midpoint-half-offset domain given by

$$
\begin{aligned}
& P\left(x_{1}, x_{2}, h_{1}=0, h_{2}=0, z, t=0\right) \\
& \quad=\iiint \iint \tilde{P}\left(x_{1}^{0}, x_{2}^{0}, h_{1}^{0}, h_{2}^{0}, z=0, \omega\right) \\
& \quad \times \exp (i \omega T) d \omega d k_{h 1} d k_{h 2} d k_{x 1} d k_{x 2},
\end{aligned}
$$


where the corresponding traveltime shift $T$ is

$$
\begin{aligned}
T & =\frac{\operatorname{DSR}\left(x_{1}, x_{2}, h_{1}, h_{2}\right)}{\omega} z-\frac{k_{x 1}}{\omega}\left(x_{1}-x_{1}^{0}\right) \\
& -\frac{k_{x 2}}{\omega}\left(x_{2}-x_{2}^{0}\right)+\frac{k_{h 1}}{\omega} h_{1}^{0}+\frac{k_{h 2}}{\omega} h_{2}^{0} .
\end{aligned}
$$

Furthermore, from equation A-17 and the relations between the midpoint-half-offset wavenumber and slowness projections (Alkhalifah, 2000b),

$$
\left(k_{x 1}, k_{x 2}\right)=\left(2 \omega p_{x 1}, 2 \omega p_{x 2}\right)
$$

and

$$
\left(k_{h 1}, k_{h 2}\right)=\left(2 \omega p_{h 1}, 2 \omega p_{h 2}\right) .
$$

The traveltime shift $T$ given in equation A-25 becomes

$$
\begin{aligned}
T & =\left(q_{s}+q_{g}\right) z-2 p_{x 1}\left(x_{1}-x_{1}^{0}\right) \\
& -2 p_{x 2}\left(x_{2}-x_{2}^{0}\right)+2 p_{h 1} h_{1}^{0}+2 p_{h 2} h_{2}^{0},
\end{aligned}
$$

where $q_{s}=k_{s z} / \omega$ and $q_{g}=k_{g z} / \omega$ are the vertical slowness components for source and receiver, respectively.

The traveltime shift $T$ given in equation A-27 can also be written in a vector form:

$$
T=\mathbf{p}_{s} \cdot\left(\mathbf{x}_{s}-\mathbf{x}\right)+\mathbf{p}_{g} \cdot\left(\mathbf{x}_{g}-\mathbf{x}\right),
$$

where $\mathbf{p}_{s}=\left(p_{x 1}-p_{h 1}, p_{x 2}-p_{h 2},-q_{s}\right)$ and $\mathbf{p}_{g}=\left(p_{x 1}+p_{h 1}\right.$, $\left.p_{x 2}+p_{h 2},-q_{g}\right)$ denote source and receiver slowness vectors, respectively; $\quad$ and $\quad \mathbf{x}_{s}=\left(x_{1}^{0}-h_{1}^{0}, x_{2}^{0}-h_{2}^{0}, 0\right), \quad \mathbf{x}_{g}=\left(x_{1}^{0}+h_{1}^{0}, x_{2}^{0}+\right.$ $\left.h_{2}^{0}, 0\right)$, and $\mathbf{x}=\left(x_{1}, x_{2}, z\right)$ denote the spatial positions of the source, receiver, and image point. In terms of the slowness projections, equation A-28 is identical to equation 4 in the main text.

For a vertically inhomogeneous anisotropic medium, the vertical slowness components $q_{s}$ and $q_{g}$ for source and receiver are depthdependent in equation A-27. In this case, traveltime shift $T$ given in equation $\mathrm{A}-27$ becomes

$$
\begin{aligned}
T & =\int_{0}^{z}\left(q_{s}(z)+q_{g}(z)\right) d z-2 p_{x 1}\left(x_{1}-x_{1}^{0}\right) \\
& -2 p_{x 2}\left(x_{2}-x_{2}^{0}\right)+2 p_{h 1} h_{1}^{0}+2 p_{h 2} h_{2}^{0} .
\end{aligned}
$$

\section{APPENDIX B}

\section{THE SIGN SELECTIONS FOR $P_{V}$ AND $Q_{V}$ IN EQUATIONS 31 AND 32}

Here, we derive the signs for $p_{v}$ and $q_{v}$ given in equations 31 and 32. From equation 4 , the one-way traveltime $t$ for a source or receiver is given by

$$
t=z q+p_{1} y_{1}+p_{2} y_{2},
$$

where the definitions of $\left(p_{1}, p_{2}, q\right)$ and $\left(y_{1}, y_{2}\right)$ are shown in the paragraphs after equations 6 and 8 , respectively.

By considering the slowness surface rotation given by equation 10 , we rewrite one-way traveltime B-1 in the form,

$$
t=\tilde{y}_{1} p_{v 1}+\tilde{y}_{2} p_{v 2}+\tilde{z} q_{v},
$$

where $\left(\tilde{y}_{1}, \tilde{y}_{2}, \tilde{z}\right)$ denotes the projection of the propagation distance $\left(y_{1}, y_{2}, z\right)$ on the VTI coordinate system,

$$
\left(\begin{array}{c}
\tilde{y}_{1} \\
\tilde{y}_{2} \\
\tilde{z}
\end{array}\right)=\left(\begin{array}{ccc}
0 & 0 & -1 \\
-\sin \phi & \cos \phi & 0 \\
\cos \phi & \sin \phi & 0
\end{array}\right)\left(\begin{array}{c}
y_{1} \\
y_{2} \\
z
\end{array}\right) .
$$

We further consider the azimuth $\alpha$ of the slowness $\left(p_{v 1}, p_{v 2}, q_{v}\right)$ in the VTI medium. We derive the expression for $\cos \alpha$ from equations 21,25 , and 27 ,

$$
\cos \alpha=\gamma \frac{z}{\sqrt{\left(y_{1} \sin \phi-y_{2} \cos \phi\right)^{2}+z^{2}}},
$$

and the expression for $\sin \alpha$ from equations 22, 26, and 27,

$$
\sin \alpha=\gamma \frac{y_{1} \sin \phi-y_{2} \cos \phi}{\sqrt{\left(y_{1} \sin \phi-y_{2} \cos \phi\right)^{2}+z^{2}}},
$$

where $r$ is given by

$$
r=\frac{y_{1} \cos \phi+y_{2} \sin \phi}{\left|y_{1} \cos \phi+y_{2} \sin \phi\right|} .
$$

From equations 23, 24, B-4, and B-5, traveltime equation B-2 becomes

$$
t=\tilde{y} p_{v}+\tilde{z} q_{v}
$$

where the horizontal and vertical propagation distances $\tilde{y}$ and $\tilde{z}$ are given by

$$
\begin{aligned}
\tilde{y} & =\tilde{y}_{1} \cos \alpha+\tilde{y}_{2} \sin \alpha \\
& =-r \sqrt{\left(y_{1} \sin \phi-y_{2} \cos \phi\right)^{2}+z^{2}}
\end{aligned}
$$

and

$$
\tilde{z}=y_{1} \cos \phi+y_{2} \sin \phi
$$

It is known that the ray direction and the slowness direction are always located in the same quadrant for a specified ray in a VTImedium (Tsvankin, 2001, pp. 22-29). It follows that $\tilde{y}(\tilde{z})$, and $p_{v}\left(q_{v}\right)$ always have the same sign. Hence, we can determine the sign for $p_{v}$ and $q_{v}$ from equations B-8 and B-9.

\section{APPENDIX C}

\section{SHANKS TRANSFORM}

Here, we use the Shanks transform to derive equations 40 and 41. The Shanks transform can be used to improve the convergence rate of a sequence given by (Bender and Orszag, 1978, pp. 369-375)

$$
A_{n}=\sum_{m=0}^{n} \alpha_{m} \zeta^{m}
$$

where $\alpha_{m}$ and $\zeta$ are real valued, and $|\zeta|<1$. When $n \rightarrow \infty, A_{n}$ approaches its limit $A_{\infty}$. The shanks transform $S\left(A_{n}\right)$ of $A_{n}$ is defined as 


$$
S\left(A_{n}\right)=\frac{A_{n+1} A_{n-1}-A_{n}^{2}}{A_{n+1}-2 A_{n}+A_{n-1}} .
$$

This transform creates a new sequence $S\left(A_{n}\right)$, which often converges more rapidly than the old sequence $A_{n}$. The sequences $S^{2}\left(A_{n}\right)=$ $S\left[S\left(A_{n}\right)\right]$ and $S^{3}\left(A_{n}\right)=S\left\{S\left(S\left(A_{n}\right)\right)\right\}$ may be even more rapidly convergent.

To improve the accuracy of the truncated Taylor expansion given in equation 31 , we define

$$
\begin{gathered}
A_{0}=-r p_{v 0}, \\
A_{1}=-r\left[p_{v 0}+p_{v 1}(2 \eta)\right],
\end{gathered}
$$

and

$$
A_{2}=-r\left[p_{v 0}+p_{v 1}(2 \eta)+p_{v 2}(2 \eta)^{2}\right]
$$

Substituting equations C-3-C-5 into equation C-2 with $n=1$, we obtain

$$
p_{v}=-r\left(p_{v 0}+\frac{2 p_{v 1}^{2} \eta}{p_{v 1}-2 p_{v 2} \eta}\right)
$$

A similar procedure applied to equation 28 results in the final expression for $q_{v}$,

$$
q_{v}=r\left(q_{v 0}+\frac{2 q_{v 1}^{2} \eta}{q_{v 1}-2 q_{v 2} \eta}\right) .
$$

\section{APPENDIX D}

\section{THE DERIVATION OF EQUATION 52}

Here, we show the derivation of equation 52. For a horizontal reflector in 3D HTI media, the incident and reflected rays for a source and receiver pair are symmetric with respect to the $z$-axis (see Figure 1). In this case, the midpoint slowness vector $\left(p_{x 1}, p_{x 2}\right)$ becomes zero. From equation 3, we obtain $\left(p_{h 1}, p_{h 2}\right)=\left(p_{g 1}, p_{g 2}\right)$. Because we will follow the approach in the section "Slowness approximation at stationary point in 3D horizontal symmetry axis media" to calculate slowness $\left(p_{g 1}, p_{g 2}\right)$, we replace $\left(p_{g 1}, p_{g 2}\right)$ by $\left(p_{1}, p_{2}\right)$ for simplicity. It follows that the reflection traveltime equation 51 is written as

$$
T=2\left(p_{1} h_{1}^{0}+p_{2} h_{2}^{0}+q z\right)
$$

where $\left(p_{1}, p_{2}\right)=\left(p_{h} \cos \beta, p_{h} \sin \beta\right)$ denotes the horizontal slowness vector for the reflected ray and $\left(h_{1}^{0}, h_{2}^{0}\right)=\left(h^{0} \cos \gamma, h^{0} \sin \gamma\right)$ and $z=\tau v_{\text {nmo }} \sqrt{1+2 \eta} / 2$ denote the half-offset and the reflector depth.

Substitution of equation 10 into equation D-1 results in

$$
T=2\left(p_{v 1} \tilde{y}_{1}+p_{v 2} \tilde{y}_{2}+q_{v} \tilde{z}\right),
$$

where $\left(\tilde{y}_{1}, \tilde{y}_{2}, \tilde{z}\right)$ denotes the projection of displacement $\left(h_{1}^{0}, h_{2}^{0}, z\right)$ in a VTI medium,

$$
\left(\begin{array}{c}
\tilde{y}_{1} \\
\tilde{y}_{2} \\
\tilde{z}
\end{array}\right)=\left(\begin{array}{ccc}
0 & 0 & -1 \\
-\sin \phi & \cos \phi & 0 \\
\cos \phi & \sin \phi & 0
\end{array}\right)\left(\begin{array}{c}
h_{1}^{0} \\
h_{2}^{0} \\
z
\end{array}\right)
$$

From equations 23 and 24 and equations B-4 and B-5, we obtain

$$
p_{v 1}=p_{v} \gamma \frac{z}{\sqrt{\left(h_{1} \sin \phi-h_{2} \cos \phi\right)^{2}+z^{2}}}
$$

and

$$
p_{v 2}=p_{v} \gamma \frac{h_{1} \sin \phi-h_{2} \cos \phi}{\sqrt{\left(h_{1} \sin \phi-h_{2} \cos \phi\right)^{2}+z^{2}}},
$$

where $r$ is given by

$$
r=\frac{h_{1}^{0} \cos \phi+h_{2}^{0} \sin \phi}{\left|h_{1}^{0} \cos \phi+h_{2}^{0} \sin \phi\right|} .
$$

In the case of an elliptical HTI medium $(\eta=0)$, the slowness expressions 40 and 41 become the exact ones,

$$
p_{v}=-\gamma \frac{c v_{0}}{v_{\mathrm{nmo}} \sqrt{c^{2} v_{0}^{2}+v_{\mathrm{nmo}}^{2}}}
$$

and

$$
q_{v 0}=\gamma \frac{v_{\mathrm{nmo}}}{v_{0} \sqrt{c^{2} v_{0}^{2}+v_{\mathrm{nmo}}^{2}}}
$$

with

$$
c=\frac{\sqrt{\left(h_{1}^{0} \sin \phi-h_{2}^{0} \cos \phi\right)^{2}+z^{2}}}{\left|h_{1}^{0} \cos \phi+h_{2}^{0} \sin \phi\right|}
$$

From these operations, traveltime equation D-2 finally becomes

$$
T=2 \sqrt{\frac{\left(h_{1}^{0} \sin \phi-h_{2}^{0} \cos \phi\right)^{2}+z^{2}}{v_{\mathrm{nmo}}^{2}}+\frac{\left(h_{1}^{0} \cos \phi+h_{2}^{0} \sin \phi\right)^{2}}{v_{0}^{2}}} .
$$

Substitution of the half-offset $\left(h_{1}^{0}, h_{2}^{0}\right)=\left(h^{0} \cos \gamma, h^{0} \sin \gamma\right)$ and the depth $z=\tau v_{\text {nmo }} / 2$ into equation D-10 leads to the P-wave reflection traveltime in a horizontal reflector in elliptical HTI media:

$$
T\left(h^{0}, \gamma\right)=\sqrt{\tau^{2}+4\left(h^{0}\right)^{2}\left(\frac{\sin ^{2}(\gamma-\phi)}{v_{\mathrm{nmo}}^{2}}+\frac{\cos ^{2}(\gamma-\phi)}{v_{0}^{2}}\right)} .
$$

\section{REFERENCES}

Alkhalifah, T., 1998, Acoustic approximations for seismic processing in transversely isotropic media: Geophysics, 63, 623-631, doi: 10.1190/1 .1444361 .

Alkhalifah, T., 2000a, An acoustic wave equation for anisotropic media: Geophysics, 65, 1239-1250, doi: 10.1190/1.1444815.

Alkhalifah, T., 2000b, The offset-midpoint traveltime pyramid in transversely isotropic media: Geophysics, 65, 1316-1325, doi: 10.1190/1 .1444823 . 
Alkhalifah, T, 2013, Traveltime approximations for inhomogeneous transversely isotropic media with a horizontal symmetry axis: Geophysical Prospecting, 61, 495-503, doi: 10.1111/j.1365-2478.2012.01067.x.

Bakulin, A., V. Grechka, and I. Tsvankin, 2000, Estimation of fracture parameters from reflection seismic data - Part I: HTI model due to a single fracture set: Geophysics, 65, 1788-1802, doi: 10.1190/1.1444863.

Bender, C. M., and S. A. Orszag, 1978, Advanced mathematical methods for scientists and engineers: McGraw-Hill.

Carcione, M., 2001, Wave fields in real media: Wave propagation in anisotropic, anelastic and porous media: Elsevier Science Publishing Company Inc.

Claerbout, J., 1985, Imaging the earth's interior: Blackwell Science, Inc.

Grechka, V., and I. Tsvankin, 1999, 3D moveout inversion in azimuthally anisotropic media with lateral velocity variation: Theory and a case study: Geophysics, 64, 1202-1218, doi: 10.1190/1.1444627.

Hao, Q., and A. Stovas, 2013, The offset-midpoint traveltime pyramid in TTI media: 75th Conference and Exhibition, EAGE, Extended Abstracts, P0105.
Shearer, P. M., and C. H. Chapman, 1988, Ray tracing in anisotropic media with a linear gradient: Geophysical Journal International, 94, 575-580, doi: 10.1111/j.1365-246X.1988.tb02277.x.

Stovas, A., and T. Alkhalifah, 2014, Mapping of moveout in a TTI medium: Geophysics, 79, no. 1, C19-C26, doi: 10.1190/geo2013-0039.1.

Thomsen, L., 1986, Weak elastic anisotropy: Geophysics, 51, 1954-1966, doi: $10.1190 / 1.1442051$.

Tsvankin, I., 2001, Seismic signatures and analysis of reflection data in anisotropic medium: Elsevier Science Publishing Company, Inc.

Tsvankin, I., and L. Thomsen, 1994, Nonhyperbolic reflection moveout in anisotropic media: Geophysics, 59, 1290-1304, doi: 10.1190/1.1443686. Yilmaz, O., 2001, Seismic data analysis, processing, inversion and interpretation of seismic data, vol. I, SEG.

Zhou, B., and S. Greenhalgh, 2008, Velocity sensitivity of seismic body waves to the anisotropic parameters of a TTI-medium: Journal of Geophysics and Engineering, 5, 245-255, doi: 10.1088/1742-2132/5/3/001. 